\title{
Are Viral Epitopes Potential Targets for Effective Glioblastoma Immunotherapy?
}

Vanessa Wilhelmi, Giuseppe Stragliotto, Cecilia Söderberg-Nauclér, and Natalia Landázuri*

Department of Medicine-Solna, Experimental Cardiovascular Research Unit, Center for Molecular Medicine, Karolinska Institute, Stockholm, Sweden

*Corresponding author: Natalia Landázuri, Ph.D. Department of Medicine-Solna, Experimental Cardiovascular Research Unit, Center for Molecular Medicine, Karolinska Institutet, Stockholm, Sweden, Tel: +46 851779844; E-mail: natalia.landazuri@ki.se

Rec date: Dec 31, 2014, Acc date: Jan 27, 2015, Pub date: Feb 04, 2015

Copyright: () 2015Wilhelmi V, et al. This is an open-access article distributed under the terms of the Creative Commons Attribution License, which permits unrestricted use, distribution, and reproduction in any medium, provided the original author and source are credited.

\begin{abstract}
Glioblastoma multiforme (GBM) is an extremely aggressive tumor with a very dismal prognosis. Although extensive efforts have been made to identify new treatment options for these patients to improve their quality of life and survival, the results are not encouraging. The patients are left in a grim situation with a short life expectancy. During the past decade, several groups have demonstrated that human cytomegalovirus (HCMV) nucleic acids and proteins can be detected in GBM tumors, while healthy brain surrounding the tumors remains negative for this virus. While a currently ongoing debate argues for or against the presence of HCMV in GBM, DC vaccinations with GBM tumor lysates have been shown to trigger an HCMV-specific immune response in patients. Moreover, HCMVspecific autologous T cells have proven capable of killing GBM cells. These observations provide immunological evidence that HCMV epitopes exist in these tumors, and support the efforts to utilize HCMV as a target for immunotherapy in GBM patients. Indeed, several HCMV-based immunotherapy trials are ongoing with encouraging results for this group of patients. Furthermore, a retrospective analysis of a cohort of GBM patients who received the anti-HCMV drug Valganciclovir as add-on to standard therapy reveals a potential substantial increase in survival. Thus, we argue that the emerging possibilities to target HCMV to substantially improve the outcome of GBM patients should be given urgent attention. In this review, we discuss potential strategies for medical or immune-based therapies to target HCMV in the context of GBM.
\end{abstract}

Keywords: Glioblastoma multiforme; Brain tumor; Chemotherapy; Radiotherapy; Immunotherapy, Cytomegalovirus

\section{Introduction}

Glioblastoma (GBM) is a highly aggressive brain tumor that affects 3-4 individuals per 100000 persons per year. It has a very dismal prognosis. Even after exhausting all the clinically approved interventions, the median survival is only 14.6 months after diagnosis [2]. Standard clinical care consists of surgical excision of the tumor, radiotherapy and chemotherapy with temozolomide as the first line of treatment [3]. Unfortunately, the borders of this tumor are diffuse and far-reaching, with tumor cells being detected at distant sites within the healthy brain. The fact that cancer cells often infiltrate brain areas that are not possible to identify or remove during surgery [4] contributes to the difficulties in finding a cure for this disease. The GBM cells that remain in the brain, or at least a subset of them, appear to be highly resistant to radiotherapy and chemotherapy. As a result, tumors recur and are lethal.

The design of effective treatments that could improve the prognosis of GBM patients and substantially increase their survival time after diagnosis has proven to be an extremely difficult task. During the past three decades, researchers around the world have devoted tremendous efforts to increase our understanding of this disease from a molecular and a pathophysiological standpoint [4]. The rather recent development of sequencing technologies capable of rapidly uncovering the cellular genome, transcriptome, proteome, metabolome and epigenetic imprint has unveiled the possibility of pinpointing molecular alterations specific to cells within tumors [3]. These alterations represent therapeutic targets that have been, and continue to be, exploited in experimental settings, with the hope of providing
GBM patients with a viable treatment. However, the conceptual design of effective therapies continues to face major barriers.

An effective therapy ideally requires to efficiently and specifically target and kill all and only cancer cells but not normal cells surrounding the tumor. One of the major difficulties derives from the fact that GBM is a heterogeneous type of tumor, with a highly distinct patient-dependent genetic and molecular imprint. As a result, GBMs are now sub classified into four different categories that have a slightly different prognosis and that are based on their gene expression profile: neural, pro-neural, classical or mesenchymal [5]. Moreover, the genetic profile can vary widely among cells within the same tumor, thereby limiting potential specific targets to a subset of cancer cells. In fact, recent data demonstrate that the four individual subtypes are present in the four different categories [6]. On the other hand, GBMs share some intrinsic characteristics that distinguish them from most (but not all) of the normal tissue. These characteristics include their high proliferative capacity, their angiogenic potency and their ability to modulate the immune system in their microenvironment to avoid recognition and killing by the immune system [7-9]. Researchers have sought to target one or a combination of the aforementioned hallmarks of GBM by designing treatments that incorporate the latest advances in biotechnology, genetic engineering, gene therapy and cell therapy [10-18]. To date, several of the proposed therapies have produced encouraging results in a preclinical setting, and have proven to be feasible and safe to patients in the clinic. Unfortunately, results from most clinical trials indicate no or minimal patient benefit.

Interestingly, and of particular relevance to the ongoing efforts to identify treatments that specifically target GBM cells, several research groups have, in the last twelve years, reported the presence of human cytomegalovirus (HCMV) proteins and nucleic acid sequences in the 
vast majority of GBMs, but not in healthy surrounding tissue [19-23] These findings, although controversial within the research community [24-26], highlight the possibility of using HCMV as a novel tumor marker and target, and raise the possibility that this virus may play a role in the pathophysiology of GBM.

Below we describe the evidence that points toward the presence of HCMV in GBM and its potential oncomodulatory role. We then describe how these findings have led to the development of investigational HCMV-targeted medical and immune therapies for GBM.

\section{Is HCMV present in GBM?}

HCMV is a double-stranded DNA virus with a genome of around 230-240 kbp. HCMV belongs to the group of $\beta$-herpesviruses. Characteristic features of this group of viruses include their speciesspecificity, a long replication cycle, the induction of cytomegaly, and the establishment of latency in certain cell types [27]. HCMV is ubiquitously spread among human populations worldwide, with a seroprevalence ranging between 50-90\%, depending on age, occupation, place of residence, sex, and socioeconomic status. Viral genes are expressed in a coordinated and regulated manner, with sequential expression of immediate-early (IE), early (E) and late (L) genes during a productive phase of infection. The two most abundantly expressed IE proteins IE72 and IE86 are important transactivators of cellular and viral genes, and are crucial for viral replication and persistence. They are encoded by the major IE gene, which consists of 5 exons and 4 introns, and can be spliced to yield different variants of the IE protein [28]. The viral pp65 protein is the most abundantly expressed tegument protein and is involved in the viral life cycle, including gene expression, assembly and egress, and it counteracts both innate and adaptive immune responses during HCMV infections [29]. IE and pp65 are two major HCMV proteins investigated in the context of GBM, and several groups report the presence of these proteins in GBM tumors.

However, the presence of HCMV in GBM tissues still remains controversial. The first report on the presence of HCMV in GBM biopsies demonstrated positive immunohistochemical (IHC) staining for the HCMV IE72 protein in 22 out of 22 samples and for the HCMV protein pp65 in 8 out of 8 samples. HCMV RNA was detected in 8 out of 8 samples, and HCMV DNA in 4 out of 4 samples. The staining was mainly tumor-cell restricted and not present in healthy tissue [19]. The presence of HCMV in GBM was subsequently both challenged [24-26] and confirmed [20-23,30,31] by other groups. The fact that recent deep-sequencing analyses failed to detect the HCMV genome in GBM samples [32,33] has further fueled the debate. Yet, in an earlier publication, HCMV DNA was detected in 11 out of 11 GBM samples by nested PCR specific for the viral major IE promoter at low and variable copy numbers [34]. In the same study, deep-sequencing analysis revealed that the tumor-associated virus strains were less variable as compared to strains in other HCMV-related diseases, which is probably due to low viral replication rates in the tumor that also result in low viral copy numbers. In another publication, multiple HCMV gene loci were analyzed by conventional and quantitative PCR analysis, and the HCMV genome was rather found in GBM samples than in other brain tumors or in epileptic brain tissue specimens [35]. Importantly, the authors emphasize the fact that the number of cellular genomes is substantially higher than the number of viral genomes, indicating that only a minority of the cells in the tumor harbor viral DNA. The low DNA copy number might be an explanation for the failure to detect viral DNA by other groups. The authors also emphasize that frozen tissue rather than paraffinembedded tissue is more suitable for the detection of viral DNA, but some of the recent deep-sequencing analyses have actually been performed on the latter kind [32]. Our analyses have confirmed the presence of HCMV DNA in very few cells in GBM tumors by in situ Fluorescence Hybridization (FISH) analysis. Generally, less than $1 \%$ of tumor cells are HCMV positive, even though a majority of tumor cells can be positive for viral proteins. These observations clearly demonstrate that the biology of HCMV in GBM tumor cells differs from the biology of this virus in normal cells. In normal cells, HCMV infection results in active replication and production of infectious virus particles and often results in cellular lysis.

Not only the presence of HCMV, but also the role of the virus in $\mathrm{GBM}$, is a matter of ongoing debate. HCMV is currently not considered to be an oncogenic virus. Yet, several features of HCMV infection such as viral immune evasion [36], promotion of angiogenesis [37], modulation of cell-signaling pathways that are implicated in cancer [38], as well as the finding that viral proteins can promote tumor formation (e.g. IE and US28) $[39,40]$ point toward an oncomodulatory role for HCMV in several cancer forms [41,42]. Further, our observations that HCMV protein expression levels in GBM tumors are of prognostic value for survival signal a potential functional association between HCMV infection of cancer cells and the development and/or progression of GBM [23]. Further investigations are hence needed to confirm previous findings and to further dissect the precise role of HCMV in GBM.

While the presence of HCMV and its role in GBM remains a topic of debate, our research group designed and conducted the VIGAS (Valcyte Treatment of Glioblastoma Patients in Sweden) phase I/II clinical trial, based on the premise that HCMV does reside in GBMs. The rationale for the study was that targeting HCMV within infected cancer cells, but not in healthy cells that are virus-negative, with the antiviral drug valganciclovir (marketed as Valcyte), could represent a new therapeutic option to treat GBM patients. Valcyte was prescribed as an add-on therapy to standard care for GBM patients with a HCMV-positive tumor (NCT00400322). Valganciclovir is an oral prodrug that converts to ganciclovir (GCV) in the intestine. GCV is phosphorylated in the cell by viral and cellular kinases to produce GCV-triphosphate, a nucleoside analog that inhibits the viral DNA polymerase and thus viral DNA synthesis. Our study demonstrated that the peroral administration of Valcyte did not cause major side effects in the patients. It is a well-tolerated drug that can be prescribed in combination with Temozolomide and radiation therapy. We observed trends, but no significant differences, in tumor volume at the time points of 3 and 6 months. We did not observe a survival benefit in patients randomized to Valcyte treatment. However, in exploratory analysis, we found a significantly higher survival rate among patients who received Valcyte for at least 6 months, regardless if they were prescribed the drug at study entry or on open label at failure or after the six-month primary end point of the study [43]. Due to the promising observations of this first trial, a number of GBM patients have requested and been prescribed Valcyte at our hospital, and been followed as an observational cohort of patients. In 2013, 50 patients had been prescribed Valcyte in our care. Forty of them, who received valganciclovir for at least 6 months, had a 2-year survival rate of $70 \%$ and a median overall survival of 30.1 months, compared to a 2-year survival rate of $18 \%$ and a median overall survival of 13.5 months in contemporary controls. Remarkably, patients who were continuously treated with valganciclovir after the first 6 months had a 2-year 
survival rate of $90 \%$ and a median overall survival of 56.4 months $[43,44]$. To date, we have treated 110 patients and are currently analyzing the outcome of their treatment.

Researchers have challenged these unprecedented results with the lack of association between positive HCMV serology of GBM patients and positive IHC staining for HCMV proteins in GBM tissue [45]. We acknowledge the observed discrepancies, and are currently investigating biological and technical aspects that could explain them. We recently reported that about $30 \%$ of GBM patients with detectable HCMV DNA, RNA and proteins in blood cells and/or in tumor specimens were HCMV seronegative based on the results from three different ELISA assays (manuscript accepted for publication). $85 \%$ of these patients had $\mathrm{T}$ cells in their blood that reacted against IE and or pp65 peptides. Thus, one biological explanation could be the presence of HCMV protein variants in GBM, such as variants of both pp65 [34] and IE proteins (unpublished data from our group) that might escape standard clinical diagnostics for HCMV serology in patients with GBM. Improvements in specificity and sensitivity of these assays might be required. However, these observations may also be explained by acquired tolerance against HCMV, or a specific loss of B cell immunity against HCMV in GBM patients.

\section{Immunotherapy concept and HCMV-targeted immunotherapy for GBM}

Despite the ongoing debate regarding the presence and role of HCMV in GBM, there have been a number of immunotherapy approaches tested to target the virus to treat GBM patients. Many of them have succeeded in specific killing of tumor cells and in improving the patients' overall survival and progression-free survival. Below we describe the concept of immunotherapy and its current implementation to target HCMV in GBM.

\section{Concept and strategies for immunotherapy in GBM patients}

Although the human body has developed potent and specific immune mechanisms to identify and destroy foreign pathogens that enter our system and threaten our health, it is quite unable to identify and remove mutated cells that form malignant tumors such as GBM. This is due, to a great extent, to the ability of tumors to create an immunosuppressive environment, for example through secretion of TGF- $\beta$ and IL-10 [7].

The concept of immunotherapy (IT) has emerged as a strategy to boost the patients' immune response against tumors, with the expectation to elicit the complex physiological chain of events that would lead to killing of cancer cells. Many of these strategies also aim at lowering the immunosuppressive state in the patients. Several IT strategies have been tested in GBM patients and shown to be feasible and safe, for example when targeting a mutant form of the epidermal growth factor receptor (EGFRvIII) that has been identified as a GBMspecific antigen. Efforts to target this and other selected GBM specific antigens have included treatment with binding peptides conjugated to exogenous molecules, vaccination and adoptive T-cell therapy $[15,46]$.

Vaccines are designed to be highly tumor-and patient specific. So far, peptides, heat-shock proteins (HSP), and dentritic cells (DC) have been used for vaccination [47]. Peptide vaccines are highly-expressed, tumor-associated antigens that are administered to the patient to prime a systemic immune response to these antigens with the aim to achieve local immunological targeting and killing of the tumor cells. In the case of HSP vaccines, tumor-associated antigens are conjugated to
HSP (usually HSP protein-complex 96) to achieve a specific and enhanced tumor response. Interestingly, HSP47 was recently described as a novel tumor-associated antigen, and T-cells specific for this antigen provided a prolonged progression-free survival and overall survival to GBM patients [48].

Efforts to target HCMV in GBM have used some of the same principles and have revolved around vaccination with DC and adoptive T-cell therapy. DC vaccination consists of isolating DC, which are professional antigen-presenting cells, from the patient and engineering them ex vivo to induce DC presentation of specific tumor antigens. Thereafter, these manipulated DC are injected into the patient with the expectation to activate tumor-specific T lymphocytes. This has been accomplished by pulsing DC with total GBM lysates that contain tumor-specific peptides for antigen presentation with $\mathrm{MHCI}$ peptides from the surface of GBMs or with mutated peptides specific to GBM and not present in healthy tissue [49-51]. It has also been achieved by pulsing of DC with tumor RNA, through direct coincubation or through DC transfection or transduction with RNA encoding for a tumor-specific antigen [52-54].

Through our experience at the Karolinska University Hospital with 10 adult patients with high grade glioma, we have collected evidence of the potential benefit of DC vaccination in selected patients. We require macroscopic tumor resection and utilize freshly frozen tumor material to prepare the vaccine. The concentration of $\mathrm{CD} 4+$ and $\mathrm{CD} 8+$ blasts, which we measured before each treatment, increased and remained at high levels after the third vaccine. Although this provides evidence that the patients have mounted an immunologic-specific response against the tumor, it does not predict survival. Recently, we modified the protocol to combine DC vaccination with valganciclovir anti-viral therapy.

In the case of adoptive T-cell therapy, $\mathrm{T}$ cells are isolated from the patient, cultured ex vivo and exposed to either DCs that have been engineered as described above to present tumor-specific antigens, or by exposure to tumor-specific peptides [55]. T cells that are activated to specifically target the tumor-associated antigen of choice are then infused into the patient.

\section{HCMV-targeted IT for GBM}

The first observation that HCMV-IT could play an important role in the treatment of GBM originated from an IT approach showing that vaccination of a GBM patient with DCs that had been pulsed with an autologous tumor lysate induced a robust $\mathrm{CD} 8+\mathrm{T}$-cell response to the pp65 immunodominant epitope of HCMV, already after the first vaccination [49]. A related HCMV-targeted IT approach was presented by the group of Dr. Mitchell [53]. They isolated DC from GBM patients, pulsed them with HCMV pp65 RNA and used them to stimulate autologous $\mathrm{T}$ cells to expand pp65-specific $\mathrm{T}$ cells. These pp65-specific $\mathrm{T}$ cells could be expanded 10- to 20 -fold in vitro and were shown to recognize and lyse autologous primary GBM tumor cells in an antigen-specific manner. Also, through activation with total tumor RNA-pulsed DC, they were able to expand HCMV-specific T cells that killed pp65-expressing target cells in vitro. These results are in perfect accordance with the above-mentioned study [49] and further support the concept of endogenous HCMV antigen expression in GBM cells. Based on these findings, the same research group is currently conducting 3 phase-I clinical trials in newly diagnosed adult GBM patients [55]. One trial aims at evaluating the feasibility and safety of vaccination with CMV pp65 RNA-pulsed DC, with or without adoptive transfer of autologous pp65-stimulated T-cells 
(NCT00639639). Another trial combines the pp65 RNA-pulsed DC vaccine with Basiliximab. It aims at evaluating if the drug inhibits recovery and functionality of regulatory $\mathrm{T}$ cells during recovery from therapeutic temozolomide-induced lymphopenia (NCT00626483). In the third trial, patients are treated with autologous pp65-stimulated Tcells, and are then vaccinated with pp65 RNA-pulsed DC. The goal is to evaluate the extent to which DC vaccination, during recovery from therapeutic temozolomide-induced lymphopenia, further enhances the $\mathrm{T}$-cell response to the tumor (NCT00693095). Early data from these studies have demonstrated the capacity to enhance HCMV-specific immune responses in patients with GBM using autologous DC vaccination and adoptive cellular therapy. These data also indicate promising progression-free survival and overall survival in patients receiving HCMV-specific immunotherapy (Duane Mitchell personal communication and manuscript under review).

The research group led by Dr. Ahmed has also pursued the generation of HCMV-specific T cells for adoptive IT in GBM patients. They reported that out of 11 patients with positive HCMV serology, $45 \%$ displayed positive IHC staining for pp 65 and 91\% for IE in tumor sections. The frequency of pp65-specific T cells was lower in GBM patients as compared to healthy donors, while the frequency of IEspecific $\mathrm{T}$ cells was comparable between both groups [56]. They expanded HCMV-specific T cells from 6 GBM patients by exposing them to antigen-presenting cells that had been transduced with an adenovirus vector encoding HCMV pp65 and IE. These T cells were capable of killing autologous HLA-matched GBM cells. Their first clinical trial to evaluate the administration of HCMV-specific cytotoxic T cells in GBM patients was terminated due to poor accrual (NCT01205334). Currently, they are conducting a clinical trial to evaluate the safety of escalating doses of autologous HCMV-specific cytotoxic T-cells that are genetically modified to express chimeric antigen receptors (CAR) targeting the HER2 molecule in patients with HER2-positive GBM, who have recurrent or progressive disease after front line therapy (NCT01109095).

The group of Dr. Khanna developed a protocol to isolate, expand, and adoptively transfer HCMV-specific T cells in patients with GBM. A functional analysis of the ex-vivo isolated $\mathrm{T}$ cells showed that a large proportion was unable to produce multiple cytokines and exerted limited cytolytic activity. However, after in-vitro stimulation with HLA-matched synthetic HCMV peptides and in-vitro expansion, adoptive transfer of these $T$ cells in combination with temozolomide therapy into a patient with recurrent GBM coincided with a long-term disease-free survival [57]. In their first phase-I trial on autologous Tcell therapy that targets HCMV in recurrent GBM (ACTRN12609000338268), they expanded HCMV-specific T cells from 13 out of 19 recurrent GBM patients, whereof 11 received up to four T-cell infusions in combination with chemotherapy. The treatment was well tolerated with minor side effects. The combined treatment resulted in a median survival of 403 days. Of interest, 4 out of 10 patients remained progression-free during the study period [58, 59]. In a subsequent review, the group reported a follow-up survival time for the 4 patients since initiation of the T-cell therapy, which ranged between 10 months and more than 4 years [60]. In 2013, they prospectively registered a phase I/II clinical trial to assess safety and tolerability of autologous HCMV-specific T-cell therapy as adjuvant treatment for GBM (ACTRN12613000838718).

The above-mentioned IT findings indicate that autologous adoptive T cell-based therapy is a safe and promising strategy to target HCMVpositive GBM as an add-on to standard therapy. Additional studies have also shown that infiltration of the brain with CD8+ T cells is associated with long-term survival of GBM patients [61]. These encouraging findings warrant further investigation to facilitate and accelerate their translation to the clinic.

\section{Opportunities for new HCMV-based strategies for GBM therapy}

Promising results from HCMV-based IT that are now being reported by several investigators present HCMV as a valid and encouraging novel target for the treatment of GBM. Alternative strategies that would indirectly target HCMV within GBM include targeting of cell-signaling pathways that are modulated by, and necessary for, HCMV infection [38]. Remarkably, most of these pathways are also altered in GBM cells, and have already been identified as therapeutic targets for this particular tumor based on their ability to support cancer-cell survival, proliferation and migration. The list of therapeutic targets, which interestingly coincide with HCMV-modulated pathways, include epidermal growth factor receptor (EGFR), platelet-derived growth factor receptor (PDGFR), Ras, and mammalian target of rapamycin (mTOR) [38].

EGFR is one of the most investigated antigen-specific targets for GBM IT. EGFR is overexpressed in about $40 \%$ of GBMs. Also, a portion of this receptor, which corresponds to deletion of exons 2-7 of the EGFR gene, is mutated in $20-30 \%$ of the tumors and designated as EGFRvIII [50]. This mutant receptor is ligand-independent constitutively active. Overexpression and mutation of this receptor lead to upregulation of the EGFR signaling pathway, which in turn leads to increased cell proliferation, migration, invasion, and inhibition of apoptosis [50]. Of interest, HCMV can utilize EGFR for viral entry into certain cells, and EGFR inhibition can prevent HCMV infection [62]. A clear example of an IT strategy targeting this receptor is Rindopepimut, an EGFRvIII-specific peptide conjugated to the exogenous carrier protein Keyhole Limpet Hemocyanin (KLH) that contains multiple epitopes to serve as an adjuvant. Rindopepimut is currently in phase 3 clinical trial, based on its ability to stimulate an immune response against EGFRvIII [63].

The angiogenic pathway has also been targeted for GBM therapy. Upregulation of vascular endothelial growth factor (VEGF) in GBM mediates a strong pro-angiogenic phenotype, which leads to the aberrant and uncontrolled growth of tumor. Interestingly, the HCMV protein US28 induces production of VEGF via nuclear factor kappa-B induced Cox-2 expression $[64,65]$. The anti-VEGF-A monoclonal antibody bevacizumab was developed as a blocking antibody that inhibits the VEGF-A functionality as a ligand, and blocks the proangiogenic cascade of events that follow upon binding of VEGF-A to its receptor. This antibody showed promising radiological results delaying tumor recurrence (although not extension in patient survival) during clinical trials, and is now approved as an angiogenesis inhibitor for the clinical treatment of GBM in the US [66] , but not in the EU.

GBM is also characterized by the expression of platelet-derived growth factor (PDGF), which mediates signaling through its receptors (PDGFR) alpha and beta to induce mitogenic and migratory responses. PDGFR alpha is necessary for HCMV entry, signaling and productive infection [67]. Blocking this receptor or inhibiting its kinase activity render target cells non-permissive to HCMV entry. Imatinib, a small-molecule inhibitor that blocks the kinase activity of PDGFR, and PDGFR-alpha blocking antibody have been tested as a treatment for GBM $[68,69]$. Interestingly, both drugs were also shown 
to block HCMV entry in vitro, in fibroblasts, endothelial cells and epithelial cells [67].

Yet another therapeutic target in the treatment of GBM is the mTOR pathway, which is upregulated and leads to cell proliferation and survival [70]. Inhibitors of this pathway are thus under investigation to treat GBM patients. As an example, everolimus reached phase II clinical trials as an add-on to standard radiotherapy and chemotherapy treatments [71]. mTOR is also activated during the late phase of HCMV infection and is necessary for viral replication [72]. Of interest, the same inhibitor, everolimus, has been shown to reduce HCMV infection in transplant recipients [73].

In conclusion, Rindopepimut, bevacizumab, imatinib, IMC-3G3 and everolimus are examples of drugs that could have a dual-targeted effect on GBM as a whole and on tumor-resident HCMV. As a result, they have the potential to provide a powerful (add-on) therapy for GBM patients.

Of further interest, dexamethasone, a corticosteroid routinely used in the clinic for the treatment of GBM can also affect HCMV-related signaling pathways. Dexamethasone is used to treat tumor-associated edema and to prevent inflammatory reactions in GBM patients. This drug is also investigated as part combination therapies in various ongoing clinical trials for GBM (NCT01866449, NCT00445965, NCT02031965). In vitro, this drug has been shown to inhibit the migration and invasion of U373MG GBM cell line through inhibition of the ERK1/2 MAPK pathway. However, it did not affect Hs683 glioma cells, which exemplifies glucocorticoid resistance in some GBMs [74]. During HCMV infection, dexamethasone has been shown to induce viral replication in epithelial cells and fibroblasts $[75,76]$. In agreement with this finding, treatment of various diseases with dexamethasone has led to opportunistic reactivation of HCMV, e.g. in cases of childhood acute lymphoblastic leukemia [77]. Thus, the use of dexamethasone for GBM patients might have to be evaluated in the context of potential HCMV reactivation in the tumor.

Taken together, the ability of these and other GBM therapies to modulate HCMV-related signaling pathways highlight the importance of evaluating these therapies in the context of HCMV-bearing cancer cells. This would provide the basis for designing combination HCMVtargeted IT for GBM. In fact, a recent review that addresses the mechanisms and efficacies of molecularly targeted therapies for recurrent GBM [78] highlights the need for patient-targeted combination therapies for GBM. The authors state that the clinical responses to most available targeted therapies are only modest, but that combination therapy leads to a better outcome than single-agent therapy, regardless of the agent [78]. They suggest that targeted therapies matched to molecular profiles of individual tumors might be more beneficial for patient outcome. This recommendation is highly relevant to HCMV in GBM. While HCMV is present in the vast majority of GBM, the percentage of cells that are virus-proteinpositive can vary greatly, adding to the heterogeneity of the tumor and the complexity of targeting it. It is likely that this virus only resides in a small subpopulation of tumor cells. Of note, we found that several stem cell markers were expressed on HCMV positive tumor cells, raising the possibility that HCMV may target tumor-initiating cells within the tumor, which would make this virus an ideal target for GBM therapy.

Finally, in addition to indirect targeting of the virus by affecting signaling pathways, another potential approach to directly target HCMV for therapeutic purposes in GBM patients is vaccination against HCMV. The development of an efficient HCMV-vaccine has been researched to prevent HCMV infection in a cancer-independent setting, and has been a struggle for several years. Currently, candidate vaccines include peptide vaccines, DNA vaccines, a dense-body vaccine, viral vectors, and attenuated virus vaccines. Some of these candidates have already entered phase-III clinical trials, with promising outcomes [79]. The use of HCMV vaccines for treatment of GBM patients might be of high therapeutic value if this virus is proven to be oncogenic or oncomodulatory.

\section{Conclusion}

In summary, several lines of evidence suggest that HCMV nucleic acids and proteins are present in GBM tumors. While this evidence remains a topic of debate, and controversies regarding the detection methods used for diagnostics of HCMV in tumors continue, targeting this virus in GBM has emerged as a promising strategy in immuneand medical-based therapies. As the treatment options for these patients are very limited, and substantial efforts to improve their prognosis and survival have failed, the findings from viral-targeted therapies need to be taken into consideration. Clinical trials are required with no further delay in order to provide solid data that confirm or dismiss HCMV as a novel target for GBM therapy. Given the apparent intricate nature of this virus in the context of GBM, clinical trial results may precede a mechanistic understanding of the HCMV biology within tumors. If the promising clinical results available today are confirmed, the patients, who are in desperate need for effective treatments, should have immediate access to HCMVbased therapies, even if our basic science understanding of the virus is still lagging.

\section{References}

1. World Health Organization. WHO Classification of Tumours. Glioblastoma. Tumours of the central nervous system. In International Agency for Research on Cancer.

2. Stupp R, Hegi ME, Mason WP, van den Bent MJ, Taphoorn MJ, et al. (2009) Effects of radiotherapy with concomitant and adjuvant temozolomide versus radiotherapy alone on survival in glioblastoma in a randomised phase III study: 5-year analysis of the EORTC-NCIC trial. Lancet Oncol 10: 459-466.

3. Bleeker FE, Molenaar RJ, Leenstra S (2012) Recent advances in the molecular understanding of glioblastoma. J Neurooncol 108: 11-27.

4. Wen PY, Kesari S (2008) Malignant gliomas in adults. N Engl J Med 359: 492-507.

5. Verhaak RG, Hoadley KA, Purdom E, Wang V, Qi Y, et al. (2010) Integrated genomic analysis identifies clinically relevant subtypes of glioblastoma characterized by abnormalities in PDGFRA, IDH EGFR, and NF1. Cancer cell 17: 98-110.

6. Patel AP, Tirosh I, Trombetta JJ, Shalek AK, Gillespie SM, et al. (2014) Single-cell RNA-seq highlights intratumoral heterogeneity in primary glioblastoma. Science 344: 1396-1401.

7. Avril T, Vauleon E, Tanguy-Royer S, Mosser J, Quillien V (2011) Mechanisms of immunomodulation in human glioblastoma. Immunotherapy 3: 42-44.

8. Das S, Marsden PA (2013) Angiogenesis in glioblastoma. N Engl J Med 369: 1561-1563.

9. Xie Q, Mittal S, Berens ME (2014) Targeting adaptive glioblastoma: an overview of proliferation and invasion. Neuro Oncol 16: 1575-1584.

10. Merchant RE, Ellison MD, Young HF (1990) Immunotherapy for malignant glioma using human recombinant interleukin-2 and activated autologous lymphocytes. A review of pre-clinical and clinical investigations. Journal of neuro-oncology 8: 173-188. 
11. Culver KW, Ram Z, Wallbridge S, Ishii H, Oldfield EH, et al. (1992) In vivo gene transfer with retroviral vector-producer cells for treatment of experimental brain tumors. Science 256: 1550-1552.

12. Stojdl DF, Lichty B, Knowles S, Marius R, Atkins H, et al. (2000) Exploiting tumor-specific defects in the interferon pathway with a previously unknown oncolytic virus. Nat Med 6: 821-825.

13. Green NK, Seymour LW (2002) Adenoviral vectors: systemic delivery and tumor targeting. Cancer Gene Ther 9: 1036-1042.

14. Aghi M, Martuza RL (2005) Oncolytic viral therapies - the clinical experience. Oncogene 24: 7802-7816.

15. Mitchell DA, Fecci PE, Sampson JH (2008) Immunotherapy of malignant brain tumors. Immunol Rev 222: 70-100.

16. Bexell D, Gunnarsson S, Tormin A, Darabi A, Gisselsson D, et al. (2009) Bone marrow multipotent mesenchymal stroma cells act as pericyte-like migratory vehicles in experimental gliomas. Mol Ther.17: 183-190.

17. Maatta AM, Samaranayake H, Pikkarainen J, Wirth T, Yla-Herttuala S (2009) Adenovirus mediated herpes simplex virus-thymidine kinase/ ganciclovir gene therapy for resectable malignant glioma. Current gene therapy 9: 356-367.

18. Russell SJ, Peng KW, Bell JC (2012) Oncolytic virotherapy. Nat Biotechnol 30: 658-670.

19. Cobbs CS, Harkins L, Samanta M, Gillespie GY, Bharara S, et al. (2002) Human cytomegalovirus infection and expression in human malignant glioma. Cancer Res 62: 3347-3350.

20. Mitchell DA, Xie W, Schmittling R, Learn C, Friedman A, et al. (2008) Sensitive detection of human cytomegalovirus in tumors and peripheral blood of patients diagnosed with glioblastoma. Neuro Oncol 10: 10-18.

21. Scheurer ME, Bondy ML, Aldape KD, Albrecht T, El-Zein R (2008) Detection of human cytomegalovirus in different histological types of gliomas. Acta Neuropathol 116: 79-86.

22. Lucas KG, Bao L, Bruggeman R, Dunham K, Specht C (2011) The detection of CMV pp65 and IE1 in glioblastoma multiforme. J Neurooncol 103: 231-238.

23. Rahbar A, Orrego A, Peredo I, Dzabic M, Wolmer-Solberg N, et al. (2013) Human cytomegalovirus infection levels in glioblastoma multiforme are of prognostic value for survival. J Clin Virol 57: 36-42.

24. Lau SK, Chen YY, Chen WG, Diamond DJ, Mamelak AN, et al. (2005) Lack of association of cytomegalovirus with human brain tumors. Mod Pathol 18: 838-843.

25. Poltermann S, Schlehofer B, Steindorf K, Schnitzler P, Geletneky K, et al. (2006) Lack of association of herpesviruses with brain tumors. J Neurovirol 12: 90-99.

26. Sabatier J, Uro-Coste E, Pommepuy I, Labrousse F, Allart S, et al. (2005) Detection of human cytomegalovirus genome and gene products in central nervous system tumours. Br J Cancer 92: 747-750.

27. Pellett PE, Roizman B (2007) The family herpesviridae: a brief introduction. (Field's Virology 5th edition edn).

28. Mocarski ES, Shenk T, Pass RF (2007) Cytomegaloviruses.

29. Kalejta RF, (2008) Tegument proteins of human cytomegalovirus. Microbiol Mol Biol Rev 72: 249-265, table of contents.

30. Libard S, Popova SN, Amini RM, Kärjä V, Pietiläinen T et al. (2014) Human cytomegalovirus tegument protein pp65 is detected in all intraand extra-axial brain tumours independent of the tumour type or grade. PLoS One 9: e108861.

31. Rahbar A, Stragliotto G, Orrego A, Peredo I, Taher C, et al. (2012) Low levels of Human Cytomegalovirus Infection in Glioblastoma multiforme associates with patient survival; -a case-control study. Herpesviridae 3: 3 .

32. Cimino PJ, Zhao G, Wang D, Sehn JK, Lewis JS Jr, et al. (2014) Detection of viral pathogens in high grade gliomas from unmapped next-generation sequencing data. Exp Mol Pathol 96: 310-315.

33. Cosset E, Petty TJ, Dutoit V, Cordey S, Padioleau I, et al. (2014) Comprehensive metagenomic analysis of glioblastoma reveals absence of known virus despite antiviral-like type I interferon gene response. Int J Cancer. 135: 1381-1389.
34. Bhattacharjee B, Renzette N, Kowalik TF (2012) Genetic analysis of cytomegalovirus in malignant gliomas. J Virol 86: 6815-6824.

35. Ranganathan P, Clark PA, Kuo JS, Salamat MS, Kalejta RF (2012) Significant association of multiple human cytomegalovirus genomic Loci with glioblastoma multiforme samples. Journal of virology 86: 854-864.

36. Noriega V, Redmann V, Gardner T, Tortorella D (2012) Diverse immune evasion strategies by human cytomegalovirus. Immunol Res 54: 140-151.

37. Caposio P, Orloff SL, Streblow DN (2011) The role of cytomegalovirus in angiogenesis. Virus Res 157: 204-211.

38. Roy S, Arav-Boger R (2014) New cell-signaling pathways for controlling cytomegalovirus replication. Am J Transplant. 14: 1249-1258.

39. Shen Y, Zhu H, Shenk T (1997) Human cytomagalovirus IE1 and IE2 proteins are mutagenic and mediate "hit-and-run" oncogenic transformation in cooperation with the adenovirus E1A proteins. Proc Natl Acad Sci U S A 94: 3341-3345.

40. Maussang D, Verzijl D, van Walsum M, Leurs R, Holl J, et al. (2006) Human cytomegalovirus-encoded chemokine receptor US28 promotes tumorigenesis. Proceedings of the National Academy of Sciences of the United States of America 103: 13068-13073.

41. Dziurzynski K, Chang SM, Heimberger AB, Kalejta RF, McGregor Dallas SR, et al. (2012) Consensus on the role of human cytomegalovirus in glioblastoma. Neuro Oncol 14: 246-255.

42. Cobbs CS (2013) Cytomegalovirus and brain tumor: epidemiology, biology and therapeutic aspects. Curr Opin Oncol 25: 682-688.

43. Stragliotto G, Rahbar A, Solberg NW, Lilja A, Taher C, et al. (2013) Effects of valganciclovir as an add-on therapy in patients with cytomegalovirus-positive glioblastoma: a randomized, double-blind, hypothesis-generating study. Int J Cancer. 133: 1204-1213.

44. Söderberg-Nauclér C, Rahbar A, Stragliotto G (2013) Survival in patients with glioblastoma receiving valganciclovir. N Engl J Med 369: 985-986.

45. Hellstrand K, Martner A, Bergström T (2013) Valganciclovir in patients with glioblastoma. N Engl J Med 369: 2066.

46. Aguilar LK, Arvizu M, Aguilar-Cordova E, Chiocca EA (2012) The spectrum of vaccine therapies for patients with glioblastoma multiforme. Curr Treat Options Oncol 13: 437-450.

47. Oh T. Sayegh ET, Fakurnejad S, Oyon D, Lamano JB, et al. (2015) Vaccine therapies in malignant glioma. Curr Neurol Neurosci Rep 15: 508.

48. Wu ZB, Cai L, Qiu C, Zhang AL, Lin SJ, et al. (2014) CTL responses to HSP47 associated with the prolonged survival of patients with glioblastomas. Neurology 82: 1261-1265.

49. Prins RM, Cloughesy TF, Liau LM (2008) Cytomegalovirus immunity after vaccination with autologous glioblastoma lysate. N Engl J Med 359: 539-541.

50. Gan HK, Kaye AH, Luwor RB (2009) The EGFRvIII variant in glioblastoma multiforme. J Clin Neurosci 16: 748-54.

51. Yu JS, Wheeler CJ, Zeltzer PM, Ying H, Finger DN, et al. (2001) Vaccination of malignant glioma patients with peptide-pulsed dendritic cells elicits systemic cytotoxicity and intracranial T-cell infiltration. Cancer research 61: 842-847.

52. Ashley DM Faiola B, Nair S, Hale LP, Bigner DD, et al. (1997) Bone marrow-generated dendritic cells pulsed with tumor extracts or tumor RNA induce antitumor immunity against central nervous system tumors. J Exp Med 186: 1177-1182.

53. Nair SK, De Leon G, Boczkowski D, Schmittling R, Xie W, et al. (2014) Recognition and killing of autologous, primary glioblastoma tumor cells by human cytomegalovirus pp65-specific cytotoxic T cells. Clin Cancer Res: 2684-2694.

54. Gust TC, Zenke M (2005) RNA transfer and its use in dendritic cellbased immunotherapy. Expert Opin Biol Ther 5: 173-181.

55. Nair SK, Sampson JH, Mitchell DA (2014) Immunological targeting of cytomegalovirus for glioblastoma therapy. Oncoimmunology 3: e29289.

56. Ghazi A, Ashoori A, Hanley PJ, Brawley VS, Shaffer DR, et al. (2012) Generation of polyclonal CMV-specific $\mathrm{T}$ cells for the adoptive immunotherapy of glioblastoma. J Immunother 35: 159-168. 
Citation: Wilhelmi V, Stragliotto G, Söderberg-Nauclér C, Landázuri N (2015) Are Viral Epitopes Potential Targets for Effective Glioblastoma Immunotherapy? . J Carcinog Mutagen 6: 214. doi:10.4172/2157-2518.1000214

Page 7 of 7

57. Crough T, Beagley L, Smith C, Jones L, Walker DG, et al. (2012) Ex vivo functional analysis, expansion and adoptive transfer of cytomegalovirusspecific T-cells in patients with glioblastoma multiforme. Immunol Cell Biol 90: 872-80.

58. Schuessler A, Smith C, Beagley L,Boyle GM, Rehan S et al. (2014) Autologous T-cell therapy for cytomegalovirus as a consolidative treatment for recurrent glioblastoma. Cancer Res 74: 3466-3476.

59. Schuessler A, Walker DG, Khanna R (2014) Cellular immunotherapy directed against human cytomegalovirus as a novel approach for glioblastoma treatment. Oncoimmunology 3: e29381.

60. Schuessler A, Walker DG, Khanna R (2014) Cytomegalovirus as a novel target for immunotherapy of glioblastoma multiforme. Front Oncol 4: 275.

61. Yang I, Tihan T, Han SJ, Wrensch MR, Wiencke J, et al. (2010) CD8+ Tcell infiltrate in newly diagnosed glioblastoma is associated with longterm survival. J Clin Neurosci 17: 1381-1385.

62. Wang X, Huong SM, Chiu ML, Raab-Traub N, Huang ES (2003) Epidermal growth factor receptor is a cellular receptor for human cytomegalovirus. Nature 424: 456-461.

63. Swartz AM, Li QJ, Sampson JH (2014) Rindopepimut: a promising immunotherapeutic for the treatment of glioblastoma multiforme. Immunotherapy 6: 679-690.

64. Reinhardt B, Schaarschmidt P, Bossert A, Lüske A, Finkenzeller G, et al. (2005) Upregulation of functionally active vascular endothelial growth factor by human cytomegalovirus. J Gen Virol 86: 23-30.

65. Maussang D, Langemeijer E, Fitzsimons CP, Stigter-van Walsum M, Dijkman R, et al. (2009) The human cytomegalovirus-encoded chemokine receptor US28 promotes angiogenesis and tumor formation via cyclooxygenase-2. Cancer Res 69: 2861-2869.

66. Gilbert MR, Dignam JJ, Armstrong TS, Wefel JS, Blumenthal DT, et al (2014) A randomized trial of bevacizumab for newly diagnosed glioblastoma. N Engl J Med 370: 699-708.

67. Soroceanu L, Akhavan A, Cobbs CS (2008) Platelet-derived growth factor-alpha receptor activation is required for human cytomegalovirus infection. Nature 455: 391-395.

68. Dresemann G (2005) Imatinib and hydroxyurea in pretreated progressive glioblastoma multiforme: a patient series. Ann Oncol 16: 1702-8.

69. Shah GD, Loizos N, Youssoufian H, Schwartz JD, Rowinsky EK (2010) Rationale for the development of IMC-3G3, a fully human immunoglobulin G subclass 1 monoclonal antibody targeting the platelet-derived growth factor receptor alpha. Cancer 116: 1018-1026.

70. Akhavan D, Cloughesy TF, Mischel PS (2010) mTOR signaling in glioblastoma: lessons learned from bench to bedside. Neuro Oncol 12: 882-889.

71. Ma DJ, Galanis E, Anderson SK, Schiff D, Kaufmann TJ, et al. (2014) A phase II trial of everolimus, temozolomide, and radiotherapy in patients with newly diagnosed glioblastoma: NCCTG N057K. Neuro Oncol .

72. Poglitsch M, Weichhart T, Hecking M, Werzowa J, Katholnig K, et al. (2012) CMV late phase-induced mTOR activation is essential for efficient virus replication in polarized human macrophages. Am J Transplant.12: 1458-1468.

73. Kobashigawa J, Ross H, Bara C, Delgado JF, Dengler T, et al. (2013) Everolimus is associated with a reduced incidence of cytomegalovirus infection following de novo cardiac transplantation. Transplant infectious disease : an official journal of the Transplantation Society 15: 150-162.

74. Piette C, Deprez M, Roger T, Noel A, Foidart JM, et al. (2009) The dexamethasone-induced inhibition of proliferation, migration, and invasion in glioma cell lines is antagonized by macrophage migration inhibitory factor (MIF) and can be enhanced by specific MIF inhibitors. J Biol Chem 284: 32483-32492.

75. Tanaka J, Ogura T, Kamiya S, Sato H, Yoshie T, et al. (1984) Enhanced replication of human cytomegalovirus in human fibroblasts treated with dexamethasone. J Gen Virol 65 : 1759-1767.

76. Tanaka J, Ogura T, Kamiya S, Yoshie T, Yabuki Y, et al. (1984) Dexamethasone enhances human cytomegalovirus replication in human epithelial cell cultures. Virology 136: 448-452.

77. Moritake H, Kamimura S, Kojima H, Shimonodan H, Harada M, et al. (2013) Cytomegalovirus retinitis as an adverse immunological effect of pulses of vincristine and dexamethasone in maintenance therapy for childhood acute lymphoblastic leukemia. Pediatric blood \& cancer 60: 329-331.

78. Lau D, Magill ST, Aghi MK (2014) Molecularly targeted therapies for recurrent glioblastoma: current and future targets. Neurosurg Focus 37: E15.

79. Plotkin S, Plachter B (2012) Cytomegalovirus vaccines. In: Cytomegaloviruses: from molecular pathogenesis to therapy. Caister Academic Press. 